\title{
Metastasis from a primary hepatic angiosarcoma to the colon: A case report and literature review
}

\author{
YUKAKO MOKUTANI, TAISHI HATA, YUICHIRO MIYAKE, HIDEKI KURODA, \\ HIDEKAZU TAKAHASHI, NAOTSUGU HARAGUCHI, JUNICHI NISHIMURA, \\ TSUNEKAZU MIZUSHIMA, HIROFUMI YAMAMOTO, YUICHIRO DOKI and MASAKI MORI \\ Department of Surgery, Gastroenterological Surgery, \\ Graduate School of Medicine, Osaka University, Suita, Osaka 565-0871, Japan
}

Received April 15, 2016; Accepted November 17, 2016

DOI: $10.3892 / \mathrm{ol} .2017 .5755$

\begin{abstract}
Primary hepatic angiosarcoma is a rare type of tumor with a poor prognosis. To the best of our knowledge, curative surgery of a metastatic gastrointestinal angiosarcoma from a hepatic angiosarcoma has not been reported previously. In the present report, a case of colonic metastasis from a primary hepatic angiosarcoma is discussed. A rapidly growing mass was identified in the liver of an 84-year-old Japanese male who underwent a subsegmentectomy of the liver. Microscopic examination determined that the mass was an angiosarcoma composed of sheets of spindle cells. Immunohistochemical studies confirmed the diagnosis with positive CD31 staining, which indicated the endothelial nature of the malignancy. A total of 14 months following surgery, the patient did not exhibit any symptoms; however, follow-up positron emission tomography and computed tomography images revealed a mass in the cecum. The patient underwent an ileocolectomy, and the microscopic and immunohistochemical findings indicated that the mass was a metastasized colorectal angiosarcoma. At a 4-year post-surgery follow-up appointment the patient was alive with no evidence of recurrence or metastasis.
\end{abstract}

\section{Introduction}

Angiosarcomas account for $\sim 1-2 \%$ of all soft tissue sarcomas (1), which are aggressive and malignant neoplasms of the endothelial-type cells that line vessel walls (1). They may occur in any region of the body, although the primary

Correspondence to: Dr Taishi Hata, Department of Surgery, Gastroenterological Surgery, Graduate School of Medicine, Osaka University, 2-2 Yamada-oka, Suita, Osaka 565-0871, Japan

E-mail: thata@gesurg.med.osaka-u.ac.jp

Abbreviations: $\mathrm{PET} / \mathrm{CT}$, positron emission tomography/computed tomography; SUV, standardized uptake value

Key words: angiosarcoma, liver, colorectal, metastasis, positron emission tomography/computed tomography sites are usually the skin, the breast, soft tissues and bone (2). Primary hepatic angiosarcomas are rare and represent $\sim 5 \%$ of all angiosarcomas (2-4). A complete resection of the primary hepatic tumor is the standard curative therapy; however, the majority of patients succumb to the disease within months (2-4). Gastrointestinal angiosarcomas are particularly rare (1), and, to the best of our knowledge, no case of a metastatic colorectal angiosarcoma from a primary hepatic angiosarcoma has been reported to date. A complete resection of primary hepatic angiosarcoma was obtained and the patient exhibited a disease-free survival rate of $>1$ year. Following metastasis of the lesion to the cecum, curative resection was performed, which led to increased survival. Positron emission tomography (PET) and computed tomography (CT) was used for the early diagnosis of the metastatic site. In the current study, a rare case of a metastatic colorectal angiosarcoma from a primary hepatic angiosarcoma is presented, with long-term patient survival following curative surgery.

\section{Case}

An asymptomatic gallbladder polyp with no evidence of malignancy was observed in an 84-year-old Japanese male using abdominal ultrasonography. A hypoechoic mass, $2 \mathrm{~cm}$ in diameter, was identified by ultrasonography in segment five of the liver in July 2011 at the Osaka University (Osaka, Japan). The patient's medical history included hypertension, diabetes and surgery for lung squamous cell carcinoma performed 11 years prior. The patient underwent intensive observation and, after three months, PET/CT revealed that the liver tumor had grown to $5.5 \mathrm{~cm}$ in diameter and the standardized uptake value (SUV) was determined to be 2.7 (Fig. 1A). However, there was no accumulation in the other organs. Based on the diagnosis of the primary malignant liver tumor, an S5 subsegmentectomy of the liver was performed (Fig. 1B) in January 2012. Light microscopic examination (magnification, x200; Olympus, Tokyo, Japan) revealed fascicles of spindle tumor cells forming hemorrhagic and focal necrosis (Fig. 1C). Angiosarcoma exhibited spindle-shaped cells, which demonstrated vascular formation in various patterns. Immunohistochemical staining (5) demonstrated that the tumor cells weakly expressed cluster of differentiation (CD)31 

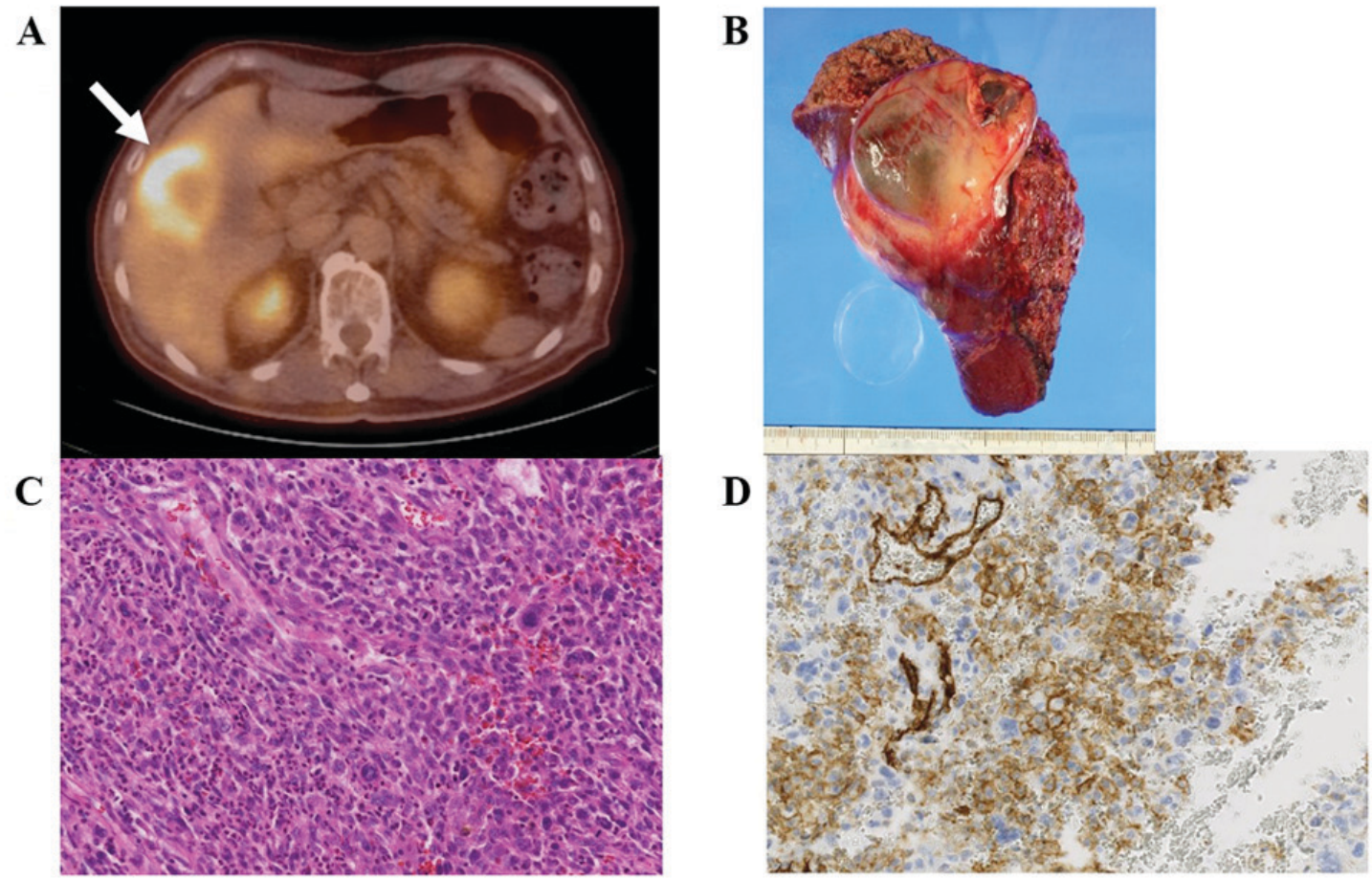

Figure 1. (A) Positron emission tomography/computed tomography revealed the uptake of the hepatic angiosarcoma. The resected angiosarcoma (B) revealed the white mass. (C) Microscopic examination (magnification, x200) following staining with hematoxylin and eosin, revealed fascicles of spindle tumor cells and (D) immunohistochemical staining (magnification, x200) demonstrated that the tumor cells weakly expressed CD31. CD, cluster of differentiation.
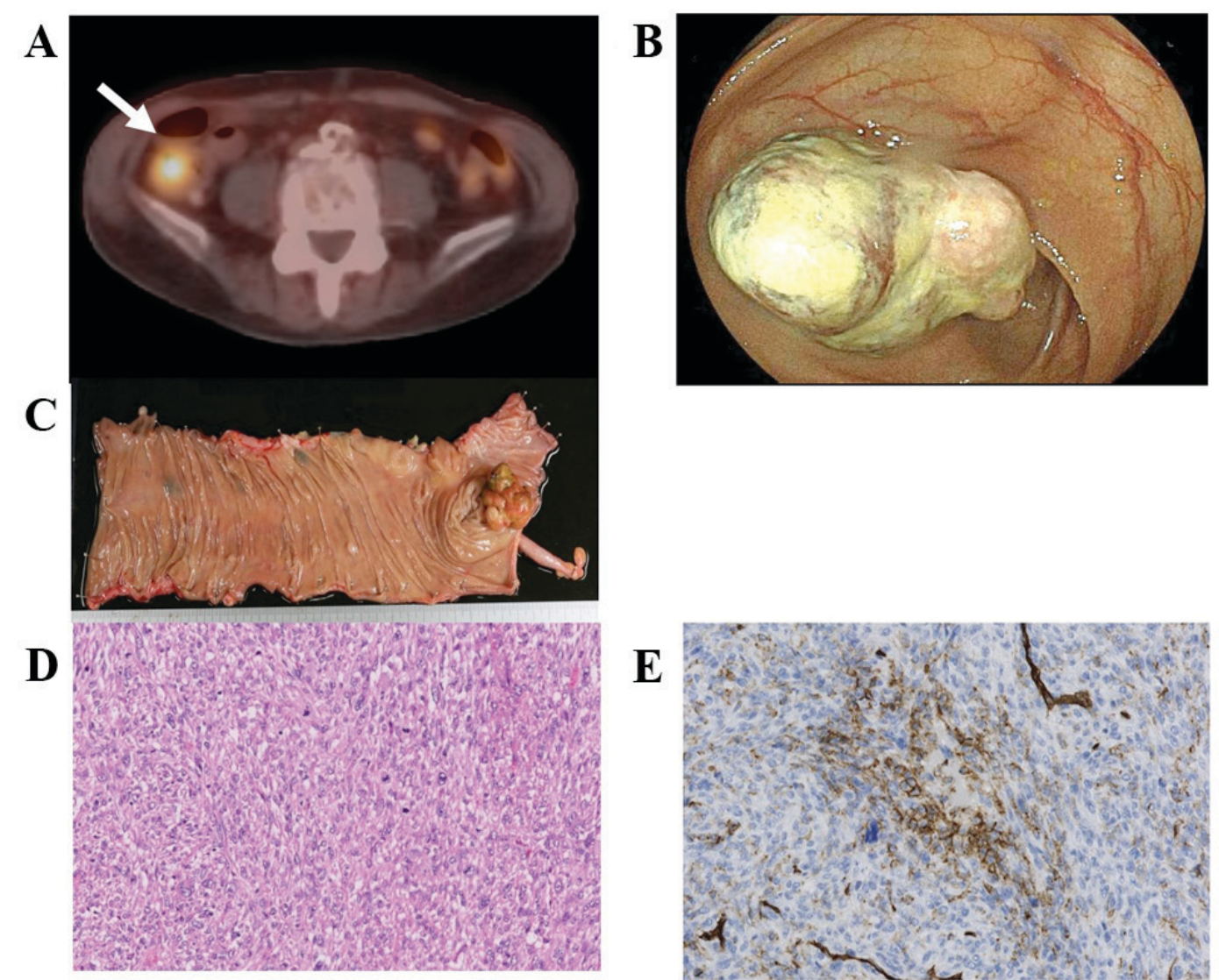

$\mathbf{E}$

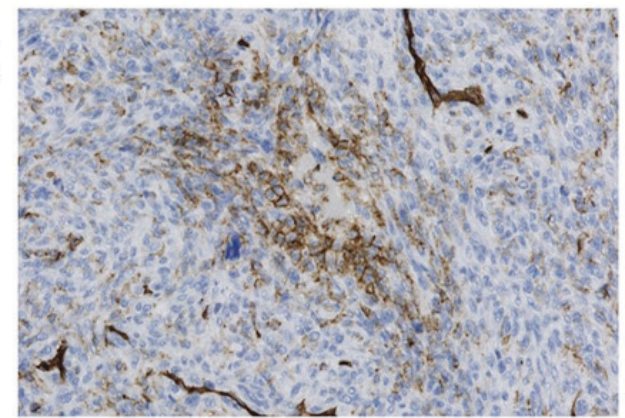

Figure 2. (A) Positron emission tomography/computed tomography of the colonic angiosarcoma. (B) The colonoscopy revealed the mass in the cecum. (C) The resected colon tissue specimen of the angiosarcoma. (D) Microscopic examination (magnification, x200) following staining with hematoxylin and eosin, and (E) immunohistochemical staining (magnification, x200) revealed the same diagnosis as for primary hepatic angiosarcoma with weak CD31 expression. $\mathrm{CD}$, cluster of differentiation. 


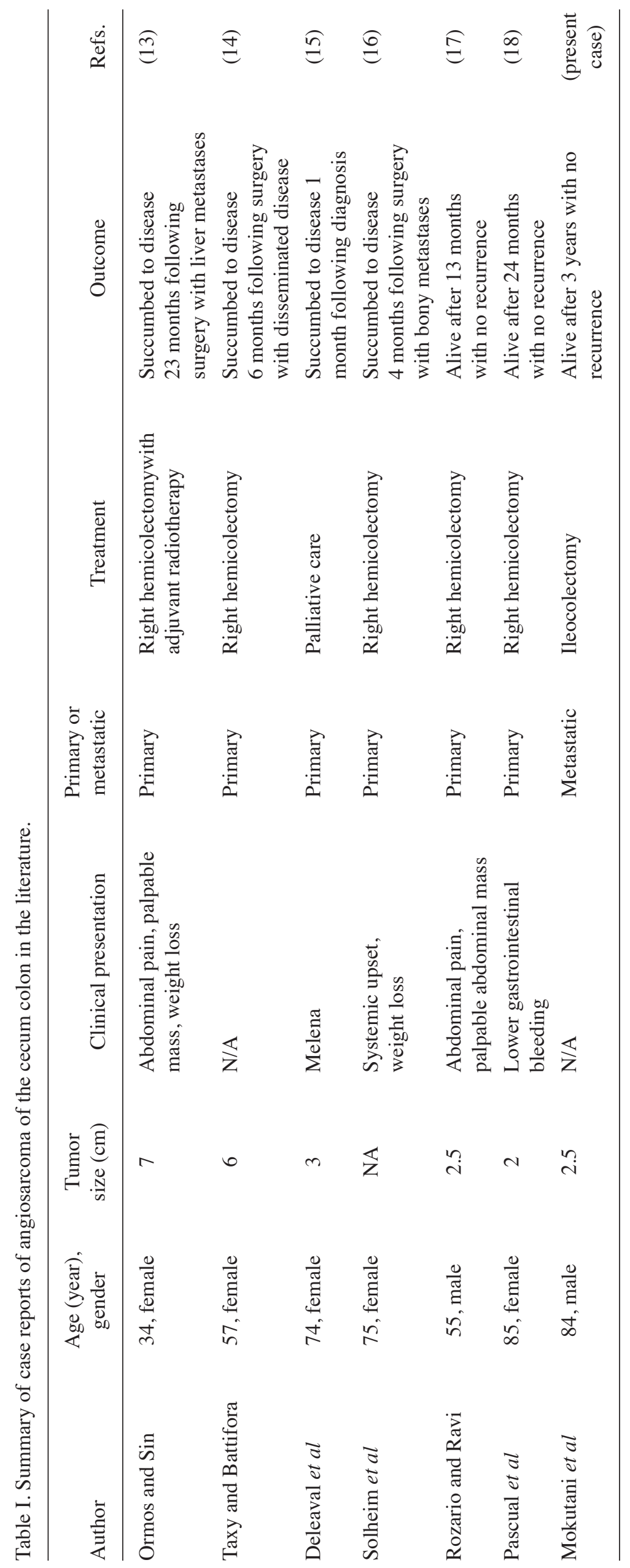


(Fig. 1D), indicating the endothelial nature of the malignancy. The tumor cells were negative for smooth muscle antigens, desmins, cytokeratins AE1/AE3 and CD34. The tumor was subsequently diagnosed as a hepatic angiosarcoma. The patient was discharged without any complications and received no adjuvant treatment following the surgery. There was no evidence of recurrence for the following 13 months. In March 2013, PET/CT indicated an SUV of $~ 9.5$ in the cecum (Fig. 2A). A colonoscopy revealed a $2.5 \mathrm{~cm}$ tumor in the cecum (Fig. 2B), and multiple biopsies suggested the diagnosis was metastatic angiosarcoma. A laparoscopic ileocolectomy was subsequently performed in May 2013 (Fig. 2C). Microscopic (magnification, x200) observations of the resected cecum tumor (Fig. 2D) and immunohistochemical staining (Fig. 2E) confirmed the diagnosis of a weakly positive CD31 primary hepatic lesion. The tumor was consistent with a metastatic colorectal angiosarcoma from a hepatic angiosarcoma. Following the second surgery, the patient received no adjuvant treatment and no evidence of recurrence was observed for a further three years. The patient was still alive four years following primary hepatic angiosarcoma surgery.

\section{Discussion}

By marked contrast with other types of soft tissue sarcoma, Fayette et al (4) reported that the overall survival of patients with angiosarcomas was influenced almost exclusively by the primary tumor site of the disease, with liver, heart, bone and splenic angiosarcomas being associated with the poorest survival time. Patients with angiosarcomas have a five-year survival of $35-45 \%(4,6)$; however, the median survival time of patients with hepatic angiosarcomas is $~ 5-6$ months $(3,7)$. It is essentially an incurable disease that is often unresectable at the time of diagnosis $(3,7)$. In addition to the current case, only a limited number of cases have been managed by partial surgical resection (3). There are a number of treatment choices, but complete surgical resection has the possibility of improving the prognosis (3,7-10). Matthaei et al (11) reported that the 5-year disease-specific survival rate was $\sim 77 \%$ for those patients who underwent complete resection of the primary hepatic sarcoma; however, the survival rate descended to $\sim 40 \%$ for those patients with primary hepatic angiosarcoma.

Angiosarcomas have a high propensity for local recurrence and distant metastases (6). Typical sites of distant metastases include the lungs, liver, regional lymph nodes, bones and skin (6). They rarely metastasize to the gastrointestinal tract, and primary or metastatic colorectal angiosarcomas are even less common, with only a few cases reported in the literature (12). There are only a few reports of cases, including the present study, that were located in the cecum (Table I). Previous studies have only focused on primary lesions and not metastasized tumors (13-18). In one of the aforementioned cases, the patient was not able to be operated upon (15). Al Beteddini et al (19) also summarized a number of colonic angiosarcoma cases and there was no metastatic operable case. To the best of our knowledge, there have been no case reports of a hepatic angiosarcoma that metastasized to the colon. In the current case, at the time of the initial surgery of the primary hepatic angiosarcoma, a screening colonoscopy was performed and no lesions were detected in the cecum.

For the primary hepatic angiosarcoma and metastatic lesion, PET/CT was useful for the detection of the tumor in the current case. An angiosarcoma does not have a consistent appearance in imaging, due to its varied histological composition (20); thus, it is challenging to differentiate an angiosarcoma from other types of vascular tumors preoperatively. Maeda et al (21) originally detailed the utility of PET/CT for the detection of primary hepatic angiosarcomas, and suggested that PET/CT may contribute to confirming the presence or absence of distant metastases in other organs, and then assist in the selection of an appropriate treatment regimen. Additionally, in the present case of metastasis to the cecum, an early diagnosis using PET/CT resulted in long-term survival and curative surgery. Although rare, the existence of metastatic colorectal angiosarcomas from primary hepatic angiosarcomas must be recognized and understood.

\section{Acknowledgements}

Written informed consent was obtained from the patient or their family for this manuscript and accompanying images. Dr Taishi HATA performed the surgery and provided substantial information regarding the patient's case. All authors have approved the final manuscript.

\section{References}

1. Weiss SW, Goldblum JR and Folpe AL: Enzinger and Weiss's soft tissue tumors. 6th edition. Elsevier Health Sciences, 2007.

2. Lahat G, Dhuka AR, Hallevi H, Xiao L, Zou C, Smith KD, Phung TL, Pollock RE, Benjamin R, Hunt KK, et al: Angiosarcoma: Clinical and molecular insights. Ann Surg 251: 1098-1106, 2010.

3. Zheng YW, Zhang XW, Zhang JL, Hui ZZ, Du WJ, Li RM and Ren XB: Primary hepatic angiosarcoma and potential treatment options. J Gastroenterol Hepatol 29: 906-911, 2014.

4. Fayette J, Martin E, Piperno-Neumann S, Le Cesne A, Robert C, Bonvalot $\mathrm{S}$, Ranchère $\mathrm{D}$, Pouillart $\mathrm{P}$, Coindre JM and Blay JY: Angiosarcomas, a heterogeneous group of sarcomas with specific behavior depending on primary site: A retrospective study of 161 cases. Ann Oncol 18: 2030-2036, 2007.

5. Orchard GE, Zelger B, Jones EW and Jones RR: An immunocytochemical assessment of 19 cases of cutaneous angiosarcoma. Histopathology 28: 235-240, 1996.

6. Mark RJ, Poen JC, Tran LM, Fu YS and Juillard GF: Angiosarcoma. A report of 67 patients and a review of the literature. Cancer 77: 2400-2406, 1996.

7. Molina $\mathrm{E}$ and Hernandez A: Clinical manifestations of primary hepatic angiosarcoma. Dig Dis Sci 48: 677-682, 2003.

8. Kobayashi T, Ishiyama K and Ohdan H: Prevention of recurrence after curative treatment for hepatocellular carcinoma. Surg Today 43: 1347-1354, 2013.

9. Amano R, Yamada N, Nakata B, Kimura K, Yashiro M, Ohira M and Hirakawa K: Prognostic indicator for the resection of liver metastasis of colorectal cancer. Surg Today 44: 1287-1292, 2014

10. Mizuguchi T, Kawamoto M, Meguro M, Hui TT and Hirata K: Preoperative liver function assessments to estimate the prognosis and safety of liver resections. Surg Today 44: 1-10, 2014.

11. Matthaei H, Krieg A, Schmelzle M, Boelke E, Poremba C, Rogiers X, Knoefel WT and Peiper M: Long-term survival after surgery for primary hepatic sarcoma in adults. Arch Surg 144: 339-344, 2009.

12. Brown CJ, Falck VG and MacLean A: Angiosarcoma of the colon and rectum: Report of a case and review of the literature. Dis Colon Rectum 47: 2202-2207, 2004.

13. Ormos J and Sin L: Hemangiosarcoma of the intestine. Zentralbl Allg Pathol 99: 352-357, 1959 (In German). 
14. Taxy JB and Battifora $\mathrm{H}$ : Angiosarcoma of the gastrointestinal tract. A report of three cases. Cancer 62: 210-216, 1988.

15. Deléaval JP, Peter MY, Laurencet F and Fontolliet C: Multicentric intestinal angiosarcoma. Report on a case. Ann Pathol 11: 342-344, 1991 (In French).

16. Solheim K, Dammen I, Roald BB and Carlsen E: Angiosarcoma of the colon. Tidsskr Nor Laegeforen 111: 3068-3069, 1991.

17. Rozario A and Ravi HR: Angiosarcoma of cecum: Unusual presentation with intussusception. Indian J Gastroenterol 14: 31-32, 1995.

18. Pascual M, Juanpere N, Alameda F and Pera M: Primary colonic angiosarcoma as a cause of massive lower gastrointestinal bleeding. Rev Esp Enferm Dig 102: 288-289, 2010.
19. Al Beteddini OS, Brenez D, Firket C, Algaba R and Tabech A: Colonic angiosarcoma: A case report and review of literature. Int J Surg Case Rep 4: 208-211, 2013.

20. Koyama T, Fletcher JG, Johnson CD, Kuo MS, Notohara K and Burgart LJ: Primary hepatic angiosarcoma: Findings at CT and MR imaging. Radiology 222: 667-673, 2002.

21. Maeda T, Tateishi U, Hasegawa T, Ojima H, Arai Y and Sugimura K: Primary hepatic angiosarcoma on coregistered FDG PET and CT images. AJR Am J Roentgenol 188: 1615-1617, 2007. 